\title{
Design and Implementation of Campus Keeping Fit Application Based on iOS and Bmob Cloud
}

\author{
Yang Liu ${ }^{1}$ \\ Dalian Neusoft Institute of Information, Computer Science and Technology \\ Liaoning Dalian, 116023, China \\ E-mail: Iiuyang_jsjeneusoft.edu.cn
}

In order to find an effective way to develop intelligent campus application and improve the science and technology level of the local campus learning and daily life, the Campus Keeping Fit application based on iOS and Bmob cloud was designed and implemented in this paper. By introducing Bmob cloud as server side, we can simplify the process of building network communication interfaces and the database management work. Then the usage of the third part libraries can make the implementation effective. For illustration, the database structure and several main functions of the intelligent campus application are introduced. The practice results show that Bmob server and whole framework used in the paper are effective and convenient way to develop intelligent campus applications. This paper designed and implemented an iOS application named Campus Keeping Fit as a demonstration to develop the intelligent campus, and it definitely can be considered as a feasible way to solve the problem of campus application designand implementation work.

\section{${ }^{1}$ Speaker}




\section{Introduction}

With the explosive growth of mobile technology, the method of campus users to obtain information has been changed. The mobile devices become more and more popular, and they gain a foothold from traditional PC on working, learning or leisure. Especially on campus, students love this more efficient and speedy way to process their school issues[1]. The efficient and speedy feature is an important point for mobile device and network to win the competition against traditional method. The campus intelligent application will be the main way to combine mobile technology and campus life [2].

This paper will describe the design process and programming technologies to implement the main functional modules which include Neu, Association, Forum and Personal Center. For the details, the application will share the information about sports activity, training master, race, and association condition in Dalian Neusoft Institute of Information to users. Meanwhile, it also supports the management service of personal information.

The client of the Campus Keeping Fit application will be built by Objective-C programming language based on iOS platform and the Xcode will be used as programming tool. In this program, Bmob cloud will play the server role which supports the data storage and transmission [3]. Compared with the traditional way to build server, it will be more efficient. The main concern of interactive application is interaction effect, so taking a simplified way to implement data server should be considered. In this paper, the application development structure of Campus Keeping Fit is expected to be helpful for all the other types of campus application implementation.

The structure of the remaining of this paper is therefore organised as follows. Section 2 shows the framework of the client/server side and the key technologies used. The design work for the whole application is mentioned in Section 3. The detailed implementation process of some main functional module are described in section 4 . Finally, in Section 5, the interpretation of the practice results are defined.

\section{Application Architecture and Key Technologies}

The Campus Keeping Fit application will be used by users in college to gather activity information and date of sports through the mobile phone. Obviously, the development of the server and client should be performed paralleled. According to the operation of users, the Client based on iOS platform will send request message to server through wireless network and wait for the replies. The iOS platform is a mobile operating system developed by Apple Inc. The Bmob network technology company provides Bmob cloud as server basic functions for free, which can handle the clients' request and respond it by the analysing data. Then the client application will show the information to users [4]. The whole process is shown in Figure1.

For the client part, several third part libraries managed by Cocoapods will be used, such as, Masonry for layout, MJRefresh for updating data, SDWebImage for loading images, and so on. And the Model-View-Controller framework will be used to specify the structure of the client application. The Bmob cloud supports numbers of interfaces for developer to manage data. The developer only needs to supply an AppKey for the application, then download Bmob SDK from its homepage and attach it into the project. 


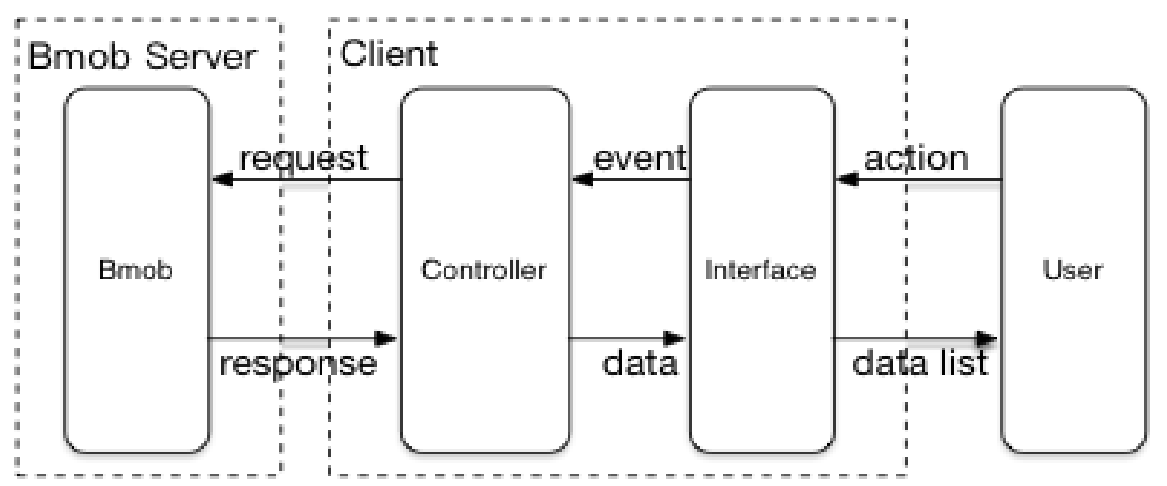

Figure 1 : the Entire Process Structure between Bmob and Users

\section{Design Details}

The main purpose of this application is to bring college students a dating sports platform which can lead them to have a healthy life style. To shoot the target, this application content will include all kinds of sports type or resources, such as, activity of association, spontaneous fitness programs and race, etc.. The application main task is to share and broadcast the sports information in the whole campus. Certainly, a free discussion zone is also built, which can attract more users to join in. In this forum, the users can post their sports pictures, exercise experiences or mood on the forum board and have conversation between the person who havethe same hobby. Finally, the personal management center is designed to implement, which is used to set custom configuration.

\subsection{Design for Server.}

Bmob cloud is friendly to developers, and it has encapsulated network request function and converts the response data to Json format for easyreading. Therefore, the developers just need to prepare the parameters and call a Bmob function during making network request. as The call back function in client will monitor the response message. During the whole data communication process, Bmob cloud can guarantee the data safety through different granularity of access control. The main job for server designing is to design the database, and the principal tables which will be set in Bmob cloud database asshown in Table 1.

\begin{tabular}{|l|l|l|}
\hline Table Name & Primary Key & \multicolumn{1}{c|}{ Description } \\
\hline User & User_id & User information, E.g. User_id, username, password, userImage, hobby \\
\hline Activity & Activity_id & Activity information. E.g. id, name, time, type, content, prefer_num, enroll_num \\
\hline Master & Master_id & Master information. E.g. id, name, type, contact, class, video \\
\hline Race & Race_id & Race information. E.g. id, name, date, detail, enroll_num \\
\hline Association & Association_id & Association information. E.g. id, name, rules, member, contact \\
\hline Forum & Forum_id & Forum information, E.g. id, title, context, image, reply \\
\hline
\end{tabular}

Table 1: Core Table List

\subsection{Design for Client.}

The whole programming structure is followed the Model-View-Controller framework. Several data models were built to store the data which client request from server, and in this 
part, the only job to do is to store the analysed Json data. The views were designed with four main storyboards as the whole application main frame and the custom cells were designed individually, in this process, the amount of properties were set to make sure the views are refreshing and friendly. The callback function will be used for reloading the real-time data. Finally, several kinds of controllers were adopted to satisfy different kinds of view styles, such as, UIViewController, UITableViewController and UICollectionViewController, and so on [5]. During the design of prototype, the professional tool Axure RP 8 is helpful. Its main framework for interfaces design is shown as Figure 2.

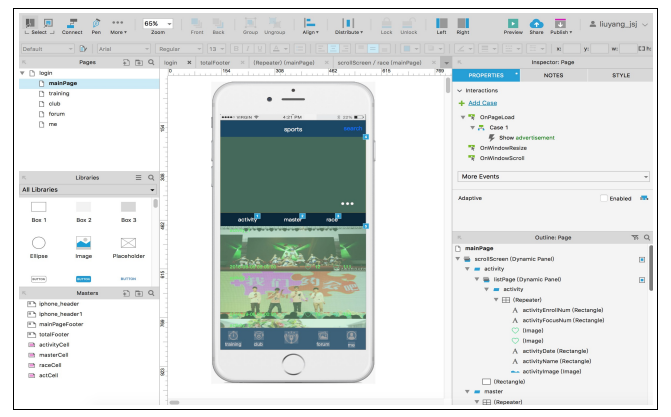

Figure 2. Axure RP 8 Design Interfaces

\section{Implementation}

The implementation of the Campus Keeping Fit application includes client base on iOS and Bmob cloud server. Because the main work of building server for this project is to set the database, and there is a detailed guide document which can be downloaded form the Bmob homepage.This paper will not mention related process again. Then, some processes of client will be introduced as sample in following paragraphs, for example, browsing activity information and signing up an activity, having an association training class.

\subsection{Activity}

To take part in kinds of activities is an effective way to spend spare life for college students. As shown in Figure 3, there is a UIScrollView appearing at the head of the main page for advertising the most popular activities, master and race. To facilitate the user's operation, the search function was supplied. The searched key words will be checked among the names or titles of events, and at the same time, the searched key words will also be sent to the Bmob Cloud server and stored as the user's hobby for making custom sports suggestion. Then the following part is a UISegmentControl widget which classifies the information embedded in the display list based on UITableView [6]. When the clicked event of the segment control occurs, the request will be sent to the Bmob Cloud server, then the related list will be reloaded. For refreshing the message list, one flag variable was created to save the page number of the sports data that is used for request new data from the Bmob Cloud. When scroll the tableview for showing information of activity, master or race, the flag variable will increase or decrease automatically to confirm the reloaded data. 


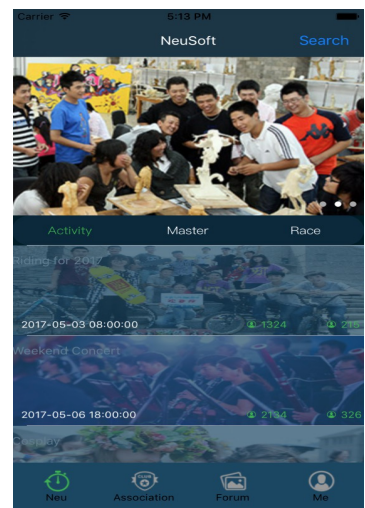

Figure 3. Main Page User Interface

Users can choose any information cell which they are interested in, then the related detailed information will show up, as shown in Figure 4. Take the activity detail page as an example, the information of the selected activity called "Riding for 2017" will be shown, such as activity name, start time, propaganda image, consulting number, and so on. If the chosen activity is the user's favourite one, it can be shared through WeChat, tencent QQ or Sina weibo [7]. The flow button located at the bottom is used for enrolling the activity. The enroll page is shown as Figure 5, and the user needs to fill his name, insurance information and purchase number to finish the enroll process.

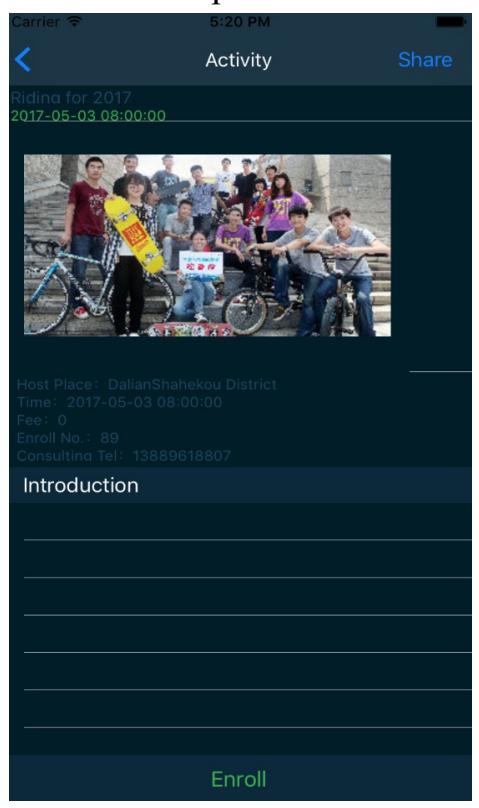

Figure 4. Activity Detail Page User Interface

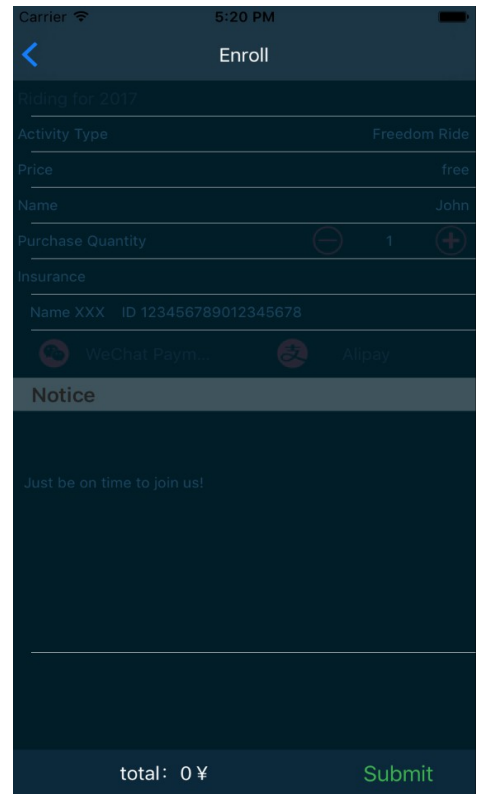

Figure 5. Activity Enroll Page User Interface

\subsection{Association.}

The main target of this modile is to advertise the information of associations and assemble more students to spend time on their interested object i. As shown in Figure 6, users can get the hottest list of all association as default or search the exact one they interest in. The search function is similar to the one mentioned in the implementation of Activity module. The "nearest" segment option can sort the association according to the geographical position of user. 
To achieve it, a positioning monitor was set when the segment changed. When any cell will be clicked, the corresponding detail page will show up as Figure 7, which includes the introduction, related video and courses. At the association detail page, users can share and follow the club which they prefer, then the related data will be updated at the server side. For the introduction of each club, the brief information, suggestion and contact way were mentioned. Users can also make appointment for the courses and watch their interested video online.

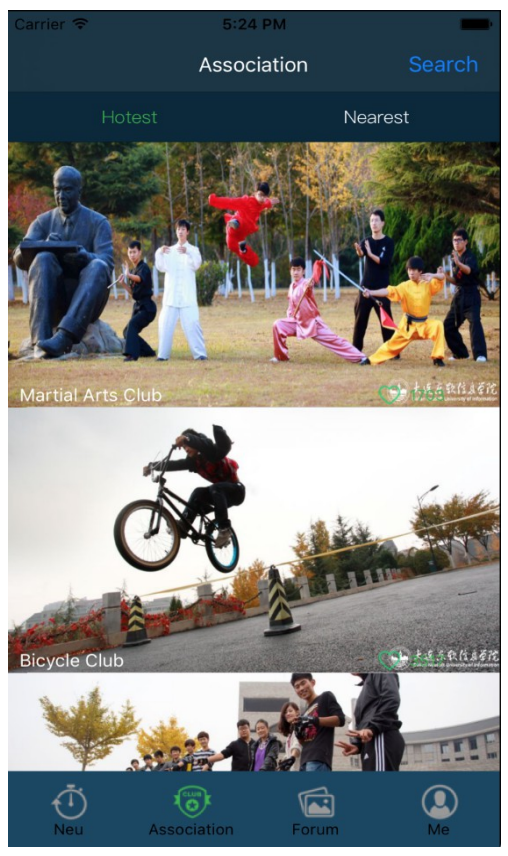

Figure 6. Association Page User Interface

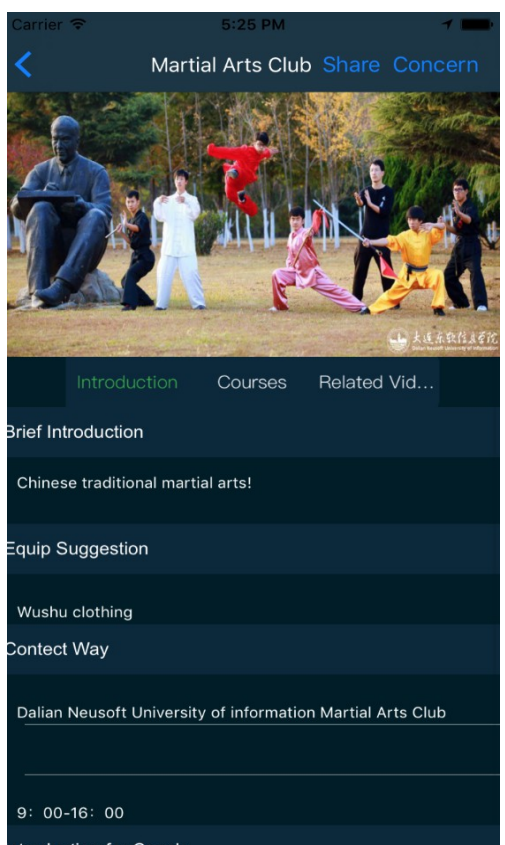

Figure 7. Association Detail Page User Interface

\section{Conclusion}

In order to diversify leisure life of college students and promote the intelligent campus development, the Campus Keeping Fit application was designed and implemented. Although due to the article length limit, there are several functional modules which have not been mentioned, the design patterns and structures can still be useful for the development of other kinds of intelligent campus applications. Especially, the Bmob cloud is a pretty good server for developers, nevertheless, the free edition has some functional limitations. The Bmob cloud server used in this paper can manage the communication data effectively and conveniently for the developers, greatly reducing the implementation difficulty compared with the traditional Web server, such as PHP or Java. For the iOS client part, the whole framework can be reused for other applications, and the common functional modules which were encapsulated well can be attached to similar applications easily.

Finally, the Campus Keeping Fit application can be a sample for developing the custom smart campus mobile applications. There are several modifications for it to supply better service for college students.

\section{References}

[1] Adrian Holzer, Jan Ondrus. Mobile application market: A developer's perspective[J]. Telematics and Information. 28 (2011) 22-31 
[2] Ndez-L, Pez, Lvaro, et al. Mobile learning technology based on iOS devices to support students with special education needs[J]. Computers \& Education, 2013, 61(5):77-90.

[3] R Zhou, Y Gao. Application of Bmob cloud platform in Android App development. Microcomputer \& Its Applications (2015)

[4] Arrigo, Marco. "Mobile formal and informal learning on the IOS platform." Edulearn11 -, International Conference on Education and New Learning Technologies 2011:6715-6722.

[5] Li A. Design and Implementation of Recording Software System Based on iOS Platform[J]. Office Informatization, 2014.

[6] Wetchakorn T, Prompoon N. Method for mobile user interface design patterns creation for iOS platform $[\mathrm{C}] / /$ International Joint Conference on Computer Science and Software Engineering. IEEE, 2015.

[7] Huang L F. Design of the Client in Wireless City Based on IOS Platform[J]. Applied Mechanics \& Materials, 2014, 556-562:5732-5735. 